\title{
The Small Time Asymptotics of SPDEs with Reflection
}

\author{
Juan Yang, ${ }^{1}$ Jianliang Zhai, ${ }^{2}$ and Qing Zhou ${ }^{1}$ \\ ${ }^{1}$ School of Science, Beijing University of Posts and Telecommunications, No. 10 Xitucheng Road, Haidian District, Beijing 100875, China \\ ${ }^{2}$ School of Mathematical Sciences, University of Science and Technology of China, Hefei, Anhui 230026, China
}

Correspondence should be addressed to Juan Yang; yangjuanyj6@gmail.com

Received 7 March 2014; Accepted 25 April 2014; Published 20 May 2014

Academic Editor: Robert A. Van Gorder

Copyright (C) 2014 Juan Yang et al. This is an open access article distributed under the Creative Commons Attribution License, which permits unrestricted use, distribution, and reproduction in any medium, provided the original work is properly cited.

\begin{abstract}
We study stochastic partial differential equations with singular drifts and with reflection, driven by space-time white noise with nonconstant diffusion coefficients under periodic boundary conditions. The existence and uniqueness of invariant measures is established under appropriate conditions. As a byproduct, the Hölder continuity of the solution is obtained. The strong Feller property is also obtained. Moreover, we show large deviation principle.
\end{abstract}

\section{Introduction}

Stochastic partial differential equations (SPDEs in short) with reflection can be used to model the evolution of random interfaces near a hard wall. Nualart and Pardoux [1] and Donati-Martin and Pardoux [2] introduced "reflection" to prevent stochastic heat equations from becoming negative, which could be viewed as an extension of one-dimensional stochastic differential equations reflected at 0 . Funaki and Olla [3] considered fluctuations around the hydrodynamical limit of a Ginzburg-Landau $\nabla \phi$ interface model on a wall. When the interface touches the wall, it will be repulsed. They proved that the fluctuations of a $\nabla \phi$ interface model near a hard wall converge in law to the stationary solution of a SPDE with reflection. They also showed that SPDEs with reflection have natural and meaningful bases in statistical mechanics, like entropic repulsion phenomena in Deuschel and Giacomin [4] and in Lebowitz and Maes [5]. There are also various models using SPDEs with reflection, such as stochastic Cahn-Hilliard equations with reflection (see da Prato and Zabczyk [6] and Debussche and Zambotti [7]) and stochastic generalized porous media equations with reflection (see Röckner et al. [8]).

In the case considered here, two reflections have been added to our model (1), and it is believed that it is a natural extension of one reflection. There are some works on this topic recently; see [9-13].

Through introducing the drift $u^{-\alpha}$, Mueller [14] gave a proof that solutions of SPDEs $u_{t}=u_{x x}+g(u) \dot{W}(t, x)$, where $g(u)$ is non-Lipschitz, do not blow up in finite time. As a byproduct, it was proved that if $\alpha>3$, this drift forces solutions to stay positive with probability 1 , and it was believed that this result "may be of interest." Then Mueller and Pardoux [15] concentrated on the case when $0<\alpha<3$ and showed that the solutions hit 0 in finite time with positive probability. Thus, the case $\alpha=3$ is the critical case for $u$ to hit zero and has been showed that it has essential relationship with reflections; for details, see Mueller [14], Mueller and Pardoux [15], and Zambotti [16, 17].

Inspired by these, our interest stays in studying SPDEs which have double smooth reflecting walls $h^{1}$ and $h^{2}$ and two singular drifts $c_{1} /\left(u-h^{1}\right)^{\alpha}$ and $c_{2} /\left(h^{2}-u\right)^{\alpha}$, for all $\alpha>0$.

For the case $\alpha=3$, it is a quite interesting topic and needs more detailed studies. As an extension from one reflection (see Zambotti $[16,17]$ ) to two-reflection case, one interesting problem is to find the explicit invariant probability measure and then study the detailed hitting properties of the solutions (see Dalang et al. [18]).

In this paper, we consider the following SPDEs:

$$
\begin{aligned}
\frac{\partial u(x, t)}{\partial t}= & \frac{\partial^{2} u(x, t)}{\partial x^{2}}+f(u(x, t)) \\
& +\frac{c_{1}}{\left(u(x, t)-h^{1}(x)\right)^{\alpha}}-\frac{c_{2}}{\left(h^{2}(x)-u(x, t)\right)^{\alpha}} \\
& +\sigma(u(x, t)) \dot{W}(x, t)+\eta(x, t)-\xi(x, t) ;
\end{aligned}
$$




$$
\begin{gathered}
u(x, 0)=u_{0}(x) \in C\left(S^{1}\right) \\
h^{1}(x) \leq u(x, t) \leq h^{2}(x), \quad \text { for }(x, t) \in Q
\end{gathered}
$$

where $Q:=S^{1} \times \mathbb{R}_{+}, \alpha \geq 0, c_{1}>0, c_{2}>0, S^{1}:=$ $\mathbb{R}(\bmod 2 \pi)$, or $\left\{e^{i \theta} ; \quad \theta \in \mathbb{R}\right\}$ denotes a circular ring and the random field $W(x, t):=W\left(\left\{e^{i \theta} ; \quad 0 \leq \theta \leq x\right\} \times[0, t]\right)$ is a regular Brownian sheet defined on a filtered probability space $\left(\Omega, P, \mathscr{F} ;\left\{\mathscr{F}_{t}\right\}_{t \geq 0}\right)$. The random forces $\xi$ and $\eta$ are added to (1) to prevent the solution from leaving the interval $\left[h^{1}, h^{2}\right]$.

We assume that the reflecting walls $h^{1}(\cdot), h^{2}(\cdot)$ are continuous functions satisfying the following.

(H1) $h^{1}(x)<h^{2}(x)$ for $x \in S^{1}$.

(H2) $\left(\partial^{2} h^{i}(x) / \partial x^{2}\right) \in L^{2}\left(S^{1}\right)$, where $\partial^{2} / \partial x^{2}$ is interpreted in a distributional sense.

We also assume that the coefficients $f, \sigma: \mathbb{R} \rightarrow \mathbb{R}$ satisfy the following:

(F1) there exists $L>0$ such that

$$
\begin{aligned}
& \left|f\left(z_{1}\right)-f\left(z_{2}\right)\right|+\left|\sigma\left(z_{1}\right)-\sigma\left(z_{2}\right)\right| \\
& \quad \leq L\left|z_{1}-z_{2}\right|, \quad z_{1}, z_{2} \in \mathbb{R}
\end{aligned}
$$

(F2) there exists $C>0$ such that

$$
|f(z)|+|\sigma(z)| \leq C(1+|z|), \quad z \in \mathbb{R}
$$

The initial condition $u_{0}(x)$ satisfies the following:

(F3) $u_{0}(x) \in C\left(S^{1}\right)$ satisfy $h^{1}(x) \leq u_{0}(x) \leq h^{2}(x)$, for $x \in$ $S^{1}$.

The following is the definition of a solution of a SPDE with two reflecting walls $h^{1}(x)$ and $h^{2}(x), x \in S^{1}$.

Definition 1. A triplet $(u, \eta, \xi)$ is a solution to the SPDE (1) if

(i) $u=\{u(x, t) ;(x, t) \in Q\}$ is a continuous, adapted random field (i.e., $u(x, t)$ is $\mathscr{F}_{t}$-measurable $\forall t \geq$ $\left.0, x \in S^{1}\right)$ satisfying $h^{1}(x) \leq u(x, t) \leq h^{2}(x)$ a.s.;

(ii) $\eta(d x, d t)$ and $\xi(d x, d t)$ are positive and adapted (i.e., $\eta(B)$ and $\xi(B)$ are $\mathscr{F}_{t}$-measurable if $\left.B \subset S^{1} \times[0, t]\right)$ random measures on $Q$ satisfying

$$
\eta\left(S^{1} \times[0, T]\right)<\infty, \quad \xi\left(S^{1} \times[0, T]\right)<\infty,
$$

for $T>0$; (iii) for all $t \geq 0$ and $\phi \in C^{\infty}\left(S^{1}\right)$, one has

$$
\begin{aligned}
& (u(t), \phi)-\left(u_{0}, \phi\right)-\int_{0}^{t}\left(u(s), \phi^{\prime \prime}\right) d s \\
& \quad-\int_{0}^{t}(f(u(s)), \phi) d s \\
& =\int_{0}^{t}\left(\frac{c_{1}}{\left(u(s)-h^{1}\right)^{\alpha}}-\frac{c_{2}}{\left(h^{2}-u(s)\right)^{\alpha}}, \phi\right) d s \\
& \quad+\int_{0}^{t} \int_{S^{1}} \phi(x) \sigma(u(x, s)) W(d x, d s) \\
& \quad+\int_{0}^{t} \int_{S^{1}} \phi(x) \eta(d x, d s) \\
& \quad-\int_{0}^{t} \int_{S^{1}} \phi(x) \xi(d x, d s), \quad \text { a.s., }
\end{aligned}
$$

where (, ) denotes the inner product in $L^{2}\left(S^{1}\right)$ and $u(t)$ denotes $u(\cdot, t)$;

(iv) we consider the following:

$$
\begin{aligned}
\int_{Q} & \left(u(x, t)-h^{1}(x)\right) \eta(d x, d t) \\
= & \int_{Q}\left(h^{2}(x)-u(x, t)\right) \xi(d x, d t)=0 .
\end{aligned}
$$

For SPDEs without reflection, the existence and uniqueness of invariant measures has been studied by many people; see Sowers [19], Mueller [20], Peszat and Zabczyk [21], and da Prato and Zabczyk [6]. For SPDEs with reflection, when the diffusion coefficient $\sigma$ is a constant, existence and uniqueness of invariant measures was obtained by Zambotti [16] and Otobe $[10,22]$, while the result was also obtained in [12] for the SPDE with a nonlinear diffusion coefficient by using coupling method. The strong Feller property of SPDEs has been studied by several authors; see Peszat and Zabczyk [21] and da Prato and Zabczyk [6]. The strong Feller property of SPDEs with reflection was proved in Zhang [23] and Yang and Zhang [12]. Moreover, with regard to the large deviations for the solution of the small noise perturbation of the equation, there exists a large amount of literature; see Dembo and Zeitouni [24] and references therein. For white noise-driven SPDEs, Sowers [19] and Cerrai and Röckner [25] set up some exponential estimates for proving large derivation principle. For SPDE with reflection, it is more efficient through the weak convergence approach; see Xu and Zhang [26] and, for the detail of this approach, the readers are referred to $[27,28]$.

All of the results mentioned above are devoted to the case of the Lipschitz coefficient. The purpose of this paper is to deal with SPDEs with reflection and singular drifts (1). The existence and uniqueness of the solution of (1) is established in [11]. We show in this paper the existence and uniqueness of invariant measures and the strong Feller property, as well as large deviation principle of (1). For the existence of invariant measures, our approach is to use the Krylov-Bogolyubov theorem. For the uniqueness, we adapted a coupling method 
used in [20]; also see [12]. The strong Feller property will be obtained by introducing a sequence of approximating solutions with the uniform strong Feller property and passing to the limit; also see [23]. For large deviation principle, we adopted weak convergence approach as in [26].

The rest of the paper is organized as follows. The existence and uniqueness of invariant measures and the Hölder continuity will be solved in Section 2. Section 3 establishes the strong Feller property. In Section 4, we deal with large deviation principle.

\section{Existence and Uniqueness of Invariant Measures}

In the beginning of this section, we present the Hölder continuity of the solution of (1) which will be used in the proof of the existence of invariant measures.

Consider the penalized problem as follows:

$$
\begin{gathered}
\frac{\partial u^{\varepsilon, \delta}}{\partial t}=\frac{\partial^{2} u^{\varepsilon, \delta}}{\partial x^{2}}+f\left(u^{\varepsilon, \delta}\right)+\sigma\left(u^{\varepsilon, \delta}\right) \cdot \dot{W}+f_{\varepsilon, \delta}\left(u^{\varepsilon, \delta}\right) ; \\
u^{\varepsilon, \delta}(x, 0)=u_{0}(x) \in C\left(S^{1}\right),
\end{gathered}
$$

where

$$
\begin{aligned}
f_{\varepsilon, \delta}(\cdot):= & \frac{\left(\arctan \left(\left[\left(\cdot-h^{1}(x)\right) \wedge 0\right]^{2}\right)\right.}{\delta} \\
& \left.+\frac{c_{1}}{\left[\delta+\left[\left(\cdot-h^{1}(x)\right) \vee 0\right]^{2}\right]^{\alpha / 2}}\right) \\
& -\left(\frac{\arctan \left(\left[\left(h^{2}(x)-\cdot\right) \wedge 0\right]^{2}\right)}{\varepsilon}\right. \\
& \left.+\frac{c_{2}}{\left[\varepsilon+\left[\left(h^{2}(x)-\cdot\right) \vee 0\right]^{2}\right]^{\alpha / 2}}\right),
\end{aligned}
$$

with $\delta, \varepsilon>0$. Notice that $f_{\varepsilon, \delta}$ is differentiable and $f_{\varepsilon, \delta}^{\prime} \leq 0$.

We need three lemmas before we present the regularity result.

Lemma 2. Suppose the hypotheses (H1), (H2), and (F1)-(F3) hold. For any $p \geq 1$ and $T>0$, there exists $\varrho>0$ such that $\sup _{0<\varepsilon, \delta<\varrho} \mathbb{E}\left(\left\|u^{\varepsilon, \delta}\right\|_{\infty}^{T}\right)^{p}<\infty\left(\|\omega\|_{\infty}^{T}:=\sup _{x \in S^{1}, t \in[0, T]}|\omega(x, t)|\right)$ and $u^{\varepsilon, \delta}$ converges uniformly on $S^{1} \times[0, T]$ to $u$ as $\varepsilon, \delta \rightarrow 0$ a.s., where $u$ and $u^{\varepsilon, \delta}$ are the solutions of (1) and the penalized SPDEs (7), respectively.

Proof. Without loss of generality, assume $c_{1}=c_{2}=1$. By $(\mathrm{H} 1)$, there exists $\delta_{0} \in(0,1)$ such that $h^{2}(x)-h^{1}(x) \geq \delta_{0}, \forall x \in S^{1}$. Set $0<\varepsilon, \delta<\delta_{0}^{2} / 2$.
Let $v^{\varepsilon, \delta}$ be the solution of equation

$$
\begin{gathered}
\frac{\partial v^{\varepsilon, \delta}(x, t)}{\partial t}=\frac{\partial^{2} v^{\varepsilon, \delta}(x, t)}{\partial x^{2}}+f\left(u^{\varepsilon, \delta}(x, t)\right) \\
+\sigma\left(u^{\varepsilon, \delta}(x, t)\right) \dot{W}(x, t) ; \\
v^{\varepsilon, \delta}(x, 0)=u_{0}(x) .
\end{gathered}
$$

Set $\bar{\Phi}^{\varepsilon, \delta}(t)=\sup _{s \leq t, y \in S^{1}}\left(v^{\varepsilon, \delta}(y, s)-\left(h^{1}(y)+h^{2}(y)-\delta_{0}\right) / 2\right)$. Note that $\bar{\Phi}^{\varepsilon, \delta}(t)$ is increasing with respect to $t$ and $v^{\varepsilon, \delta}(x, t)-$ $\bar{\Phi}^{\varepsilon, \delta}(t) \leq\left(h^{1}(x)+h^{2}(x)-\delta_{0}\right) / 2$. From analogue method as the proof in Proposition 3.1 in [11], $\bar{z}^{\varepsilon, \delta}(x, t):=v^{\varepsilon, \delta}(x, t)-$ $\bar{\Phi}^{\varepsilon, \delta}(t)-u^{\varepsilon, \delta}(x, t)$ is a solution of equation

$$
\begin{gathered}
\frac{\partial \bar{z}^{\varepsilon, \delta}}{\partial t}+\frac{\partial \bar{\Phi}^{\varepsilon, \delta}}{\partial t}=\frac{\partial^{2} \bar{z}^{\varepsilon, \delta}}{\partial x^{2}}-f_{\varepsilon, \delta}\left(u^{\varepsilon, \delta}\right) ; \\
\bar{z}^{\varepsilon, \delta}(x, 0)=0 .
\end{gathered}
$$

Assume $\left(\bar{z}^{\varepsilon, \delta}\right)^{+}(x, t)>0$; we have

$$
\begin{aligned}
& 0<\left(\bar{z}^{\varepsilon, \delta}\right)^{+}(x, t) \Longleftrightarrow \\
& 0<v^{\varepsilon, \delta}(x, t)-\bar{\Phi}^{\varepsilon, \delta}(t)-u^{\varepsilon, \delta}(x, t) \\
& \leq v^{\varepsilon, \delta}(x, t)-\left(v^{\varepsilon, \delta}(x, t)-\frac{h^{1}(x)+h^{2}(x)-\delta_{0}}{2}\right) \\
& \quad-u^{\varepsilon, \delta}(x, t) \\
&=\frac{h^{1}(x)+h^{2}(x)-\delta_{0}}{2}-u^{\varepsilon, \delta}(x, t) ;
\end{aligned}
$$

hence

$$
u^{\varepsilon, \delta}(x, t) \leq h^{2}(x)
$$

If $\left(\bar{z}^{\varepsilon, \delta}\right)^{+}(x, t)>0$ and $u^{\varepsilon, \delta}(x, t) \leq h^{1}(x)$, then, by (12),

$$
\begin{aligned}
\delta+ & {\left[\left(u^{\varepsilon, \delta}(x, t)-h^{1}(x)\right) \vee 0\right]^{2}=\delta } \\
& \leq \varepsilon+\delta_{0}^{2} \\
& \leq \varepsilon+\left[\left(h^{2}(x)-u^{\varepsilon, \delta}(x, t)\right) \vee 0\right]^{2} .
\end{aligned}
$$


On the other hand, if $\left(\bar{z}^{\varepsilon, \delta}\right)^{+}(x, t)>0$ and $u^{\varepsilon, \delta}(x, t)>h^{1}(x)$, then, by (11) and (12),

$$
\begin{aligned}
\varepsilon+ & {\left[\left(h^{2}(x)-u^{\varepsilon, \delta}(x, t)\right) \vee 0\right]^{2} } \\
& -\left(\delta+\left[\left(u^{\varepsilon, \delta}(x, t)-h^{1}(x)\right) \vee 0\right]^{2}\right) \\
= & \varepsilon-\delta+\left[h^{2}(x)-u^{\varepsilon, \delta}(x, t)\right]^{2} \\
& -\left[u^{\varepsilon, \delta}(x, t)-h^{1}(x)\right]^{2} \\
= & \varepsilon-\delta+\left[h^{2}(x)-h^{1}(x)\right] \\
& \times\left[h^{2}(x)+h^{1}(x)-2 u^{\varepsilon, \delta}(x, t)\right] \\
\geq & \varepsilon-\delta+\delta_{0}^{2} \\
\geq & \frac{\delta_{0}^{2}}{2} .
\end{aligned}
$$

Hence combining (13) and (14), $\left(\bar{z}^{\varepsilon, \delta}\right)^{+}(x, t)>0$ implies

$$
\begin{aligned}
\delta+ & {\left[\left(u^{\varepsilon, \delta}(x, t)-h^{1}(x)\right) \vee 0\right]^{2} } \\
& \leq \varepsilon+\left[\left(h^{2}(x)-u^{\varepsilon, \delta}(x, t)\right) \vee 0\right]^{2} .
\end{aligned}
$$

By (12) and (15), multiplying (10) by $\left(\bar{z}^{\varepsilon, \delta}\right)^{+}$, we have

$$
\begin{gathered}
\frac{\arctan \left(\left[\left(h^{2}(x)-u^{\varepsilon, \delta}(x, t)\right) \wedge 0\right]^{2}\right)}{\varepsilon}\left(\bar{z}^{\varepsilon, \delta}\right)^{+}(x, t) d t=0, \\
{\left[\frac{1}{\left[\delta+\left[\left(u^{\varepsilon, \delta}(x, t)-h^{1}(x)\right) \vee 0\right]^{2}\right]^{\alpha / 2}}\right.} \\
\left.-\frac{1}{\left[\varepsilon+\left[\left(h^{2}(x)-u^{\varepsilon, \delta}(x, t)\right) \vee 0\right]^{2}\right]^{\alpha / 2}}\right] \\
\times\left(\bar{z}^{\varepsilon, \delta}\right)^{+}(x, t) d t \geq 0 .
\end{gathered}
$$

Combining the fact that $\bar{\Phi}^{\varepsilon, \delta}(t)$ is increasing with respect to $t$, we have $\left(\bar{z}^{\varepsilon, \delta}\right)^{+}=0$. Hence

$$
u^{\varepsilon, \delta}(x, t) \geq v^{\varepsilon, \delta}(x, t)-\bar{\Phi}^{\varepsilon, \delta}(x, t) .
$$

Similarly, setting $\bar{z}^{\varepsilon, \delta}(x, t)=u^{\varepsilon, \delta}(x, t)-v^{\varepsilon, \delta}(x, t)-$ $\sup _{s \leq t, y \in S^{1}}\left(\left(h^{1}(y)+h^{2}(y)-\delta_{0}\right) / 2-v^{\varepsilon, \delta}(y, s)\right)$, we can show that

$$
\begin{aligned}
u^{\varepsilon, \delta}(x, t) \leq & v^{\varepsilon, \delta}(x, t) \\
& +\sup _{s \leq t, y \in S^{1}}\left(\frac{h^{1}(y)+h^{2}(y)-\delta_{0}}{2}-v^{\varepsilon, \delta}(y, s)\right) .
\end{aligned}
$$

Using a similar proof in Donati-Martin and Pardoux [2], it can be shown that $\sup _{\varepsilon, \delta} \mathbb{E}\left(\left\|v^{\varepsilon, \delta}\right\|_{\infty}^{T}\right)^{p}<\infty$; hence the inequalities (17) and (18) imply

$$
\sup _{\varepsilon, \delta} \mathbb{E}\left(\left\|u^{\varepsilon, \delta}\right\|_{\infty}^{T}\right)^{p}<\infty
$$

Since $u^{\varepsilon, \delta}$ is increasing as $\delta \downarrow 0$ by the comparison theorem of SPDEs (see Theorem 2.1 in [2]), we can show $u^{\varepsilon}:=\lim _{\delta \downarrow 0} u^{\varepsilon, \delta}$ exists a.s. and $u^{\varepsilon}$ solves

$$
\begin{aligned}
\frac{\partial u^{\varepsilon}(x, t)}{\partial t}= & \frac{\partial^{2} u^{\varepsilon}(x, t)}{\partial x^{2}}+f\left(u^{\varepsilon}(x, t)\right) \\
& +\sigma\left(u^{\varepsilon}(x, t)\right) \dot{W}(x, t) \\
& +\left(\eta^{\varepsilon}(x, t)+\frac{1}{\left(u^{\varepsilon}(x, t)-h^{1}(x)\right)^{\alpha}}\right) \\
& -\left(\frac{\arctan \left(\left[\left(h^{2}(x)-u^{\varepsilon}(x, t)\right) \wedge 0\right]^{2}\right)}{\varepsilon}\right. \\
& \left.+\frac{1}{\left[\varepsilon+\left[\left(h^{2}(x)-u^{\varepsilon}(x, t)\right) \vee 0\right]^{2}\right]^{\alpha / 2}}\right) ;
\end{aligned}
$$

$$
u^{\varepsilon}(x, 0)=u_{0}(x)
$$

where $\eta^{\varepsilon}(d x, d t):=\lim _{\delta \downarrow 0}\left(\left(u^{\varepsilon, \delta}(x, t)-h^{1}(x)\right)^{+} / \delta\right) d x d t$. By Lemma 3.1 and Remark 3.1 of [11], we know that $u^{\varepsilon}$ is decreasing as $\varepsilon \downarrow 0$. Let $v^{\varepsilon}$ be the solution of (9) replacing $u^{\varepsilon, \delta}$ by $u^{\varepsilon}$. Setting $\bar{z}^{\varepsilon}(x, t)=u^{\varepsilon}(x, t)-v^{\varepsilon}(x, t)-\sup _{s \leq t, y \in S^{1}}\left(\left(h^{1}(y)+\right.\right.$ $\left.\left.h^{2}(y)-\delta_{0}\right) / 2-v^{\varepsilon}(y, s)\right)$, similar to (18), we can show

$$
\begin{aligned}
& u^{\varepsilon}(x, t) \leq v^{\varepsilon}(x, t) \\
& +\sup _{s \leq t, y \in S^{1}}\left(\frac{h^{1}(y)+h^{2}(y)-\delta_{0}}{2}-v^{\varepsilon}(y, s)\right) .
\end{aligned}
$$

In addition, by the fact that $u^{\varepsilon}(x, t) \geq h^{1}(x), u:=\lim _{\varepsilon \downarrow 0} u^{\varepsilon}=$ $\lim _{\varepsilon \downarrow 0} \lim _{\delta \downarrow 0} u^{\varepsilon, \delta}$ exists a.s., and we can show $u$ is the solution of (1); see [11].

The continuity of $u$ can be proved similarly as in Theorem 4.1 of [2]. The uniform convergence of $u^{\varepsilon, \delta}$ with respect to $(x, t)$ follows from Dini's theorem.

Recall the following lemma from [29].

Lemma 3. Let $S \in C^{\alpha, \beta}\left(S^{1} \times[0, T]\right)$ satisfying

$$
|S(x, t)-S(y, s)| \leq C_{S}\left(|x-y|^{\alpha}+|t-s|^{\beta}\right) .
$$


Then, for $\rho_{1}, \rho_{2}>0$, there exists $S^{\rho_{1}, \rho_{2}} \in C^{\infty}\left(S^{1} \times[0, T]\right)$ such that

$$
\begin{gathered}
\left\|S^{\rho_{1}, \rho_{2}}-S\right\|_{\infty}^{T} \leq C_{\alpha, \beta} C_{S}\left(\rho_{1}^{\alpha}+\rho_{2}^{\beta}\right), \\
\left\|\frac{\partial S^{\rho_{1}, \rho_{2}}}{\partial x}\right\|_{\infty}^{T} \leq C_{\alpha, \beta} C_{S} \rho_{1}^{\alpha-1}, \\
\left\|\frac{\partial S^{\rho_{1}, \rho_{2}}}{\partial t}\right\|_{\infty}^{T} \leq C_{\alpha, \beta} C_{S} \rho_{2}^{\beta-1},
\end{gathered}
$$

where $C_{S}, C_{\alpha, \beta}$ are constants only depending on $S$ and $\alpha, \beta$, respectively.

The following result is stated in [29].

Lemma 4. Let $V \in C^{1,2}\left(S^{1} \times[0, T]\right)$ and $\Psi, F \in C\left(S^{1} \times[0, T]\right)$ with $\Psi \leq 0 ; V$ solves the equation with the homogeneous Dirichlet or Neumann boundary as follows:

$$
\begin{gathered}
\frac{\partial V}{\partial t}=\frac{\partial^{2} V}{\partial x^{2}}+\Psi V+\Psi F \\
V(x, 0)=0 ;
\end{gathered}
$$

then $\|V\|_{\infty}^{T} \leq\|F\|_{\infty}^{T}$.

Theorem 5. Let $u$ be the solution of (1). Denote

$$
\bar{u}(x, t)=u(x, t)-\int_{S^{1}} G_{t}(x, y) u_{0}(y) d y .
$$

Here $G_{t}(x, y)$ is the fundamental solution of the heat equation on $S^{1}$. Then, for any $\vartheta \in(0,1)$, there exists a finite random variable $\gamma_{\vartheta}$, which is independent of $u_{0}(x)$, such that, for $x, y \in$ $S^{1}, t, s \in[0, T]$,

$$
|\bar{u}(x, t)-\bar{u}(y, s)| \leq \gamma_{\vartheta}\left(|x-y|^{9 / 2}+|t-s|^{9 / 4}\right),
$$

and, for any $p \geq 1$,

$$
\mathbb{E} \gamma_{\vartheta}^{p}<\infty
$$

Moreover, if $u_{0} \in C^{\beta}\left(S^{1}\right), \beta \in(0,1 / 2)$, then, for $0<\omega<$ $\beta$, there exists a finite random variable $\gamma_{u}^{\omega}$, which is dependent on $u_{0}(x)$, such that

$$
|u(x, t)-u(y, s)| \leq \gamma_{u}^{\varpi}\left(|x-y|^{\varpi}+|t-s|^{\varpi / 2}\right),
$$

for $x, y \in S^{1}, t, s \in[0, T]$, and for any $p \geq 1$,

$$
\mathbb{E}\left[\gamma_{u}^{\omega}\right]^{p}<\infty
$$

Note. $C^{\beta}\left(S^{1}\right)$ denote the space of Hölder continuous functions on $S^{1}$ with Hölder exponent $\beta$, equipped with the norm

$$
\|u\|_{\beta}:=\sup _{x \in S^{1}}|u(x)|+\sup _{x, y \in S^{1} ; x \neq y} \frac{|u(x)-u(y)|}{|x-y|^{\beta}} .
$$

Proof. The scheme of verifying inequality (26) is similar to that in [29]. For reader's convenience, we write the proof in detail.

Define the stochastic convolution as follows:

$$
\begin{aligned}
S_{t}^{\varepsilon, \delta}(x):= & \int_{0}^{t} \int_{S^{1}} G_{t-s}(x, y) f\left(u^{\varepsilon, \delta}(y, s)\right) d y d s \\
& +\int_{0}^{t} \int_{S^{1}} G_{t-s}(x, y) \sigma\left(u^{\varepsilon, \delta}(y, s)\right) W(d y, d s) .
\end{aligned}
$$

Here $u^{\varepsilon, \delta}$ is the solution of (7).

By Lemma 2 and using similar arguments as Lemma 2.1 of [29], we have that, for any $\vartheta \in(0,1)$, there exists a random variable $C_{\varepsilon, \delta}$ such that, for any $s, t \in[0, T], x, y \in S^{1}$,

$$
\left|S_{t}^{\varepsilon, \delta}(x)-S_{s}^{\varepsilon, \delta}(y)\right| \leq C_{\varepsilon, \delta}\left(|x-y|^{9 / 2}+|t-s|^{9 / 4}\right),
$$

and for any $p \geq 1$

$$
\sup _{\varepsilon, \delta} \mathbb{E}\left(C_{\varepsilon, \delta}\right)^{p}<\infty
$$

Let $\bar{u}^{\varepsilon, \delta}(x, t)=u^{\varepsilon, \delta}(x, t)-\int_{S^{1}} G_{t}(x, y) u_{0}(y) d y$, and resolve $\bar{u}^{\varepsilon, \delta}$ into determine part and stochastic part; that is,

$$
\bar{u}^{\varepsilon, \delta}(x, t)=z^{S^{\varepsilon, \delta}}(x, t)+S_{t}^{\varepsilon, \delta}(x),
$$

where $z^{\mathcal{S}^{\varepsilon, \delta}}$ is the unique solution of the following PDE:

$$
\begin{gathered}
\frac{\partial z^{S^{\varepsilon, \delta}}}{\partial t}=\frac{\partial^{2} z^{S^{\varepsilon, \delta}}}{\partial x^{2}}+\bar{f}_{\varepsilon, \delta}\left(z^{S^{\varepsilon, \delta}}+S^{\varepsilon, \delta}\right) ; \\
z^{S^{\varepsilon, \delta}}(x, 0)=0,
\end{gathered}
$$

and $S^{\varepsilon, \delta}$ is defined in $(31), \bar{f}_{\varepsilon, \delta}(\cdot)=f_{\varepsilon, \delta}\left(\cdot+\int_{S^{1}} G_{t}(x, y)\right.$ $\left.u_{0}(y) d y\right)$. Let $S^{\rho_{1}, \rho_{2}} \in C^{\infty}\left(S^{1} \times[0, T]\right)$, which depends on $\rho_{1}, \rho_{2}$ and will be determined later. Denote $z^{S^{\rho_{1}, \rho_{2}}}$ by the unique solution of (35) replacing $S^{\varepsilon, \delta}$ by $S^{\rho_{1}, \rho_{2}}$.

As similar proof in Proposition 3.1 in [11], we obtain

$$
\left\|z^{S^{\varepsilon, \delta}}-z^{S^{\rho_{1}, \rho_{2}}}\right\|_{\infty}^{T} \leq\left\|S^{\varepsilon, \delta}-S^{\rho_{1}, \rho_{2}}\right\|_{\infty}^{T} .
$$

Also, Lemma 2 implies that

$$
\left\|S^{\varepsilon, \delta}-S^{\rho_{1}, \rho_{2}}\right\|_{\infty}^{T} \leq C_{\vartheta / 2} C_{\varepsilon, \delta}\left(\rho_{1}{ }^{9 / 2}+\rho_{2}{ }^{9 / 4}\right) .
$$

And so

$$
\left\|z^{S}-z^{S^{\rho_{1}, \rho_{2}}}\right\|_{\infty}^{T} \leq C_{\vartheta / 2} C_{\varepsilon, \delta}\left(\rho_{1}{ }^{\vartheta / 2}+\rho_{2}{ }^{\vartheta / 4}\right) .
$$

Differentiating $z$ in (35), $\widetilde{z}\left(:=\partial_{x} z^{S^{\rho_{1}, \rho_{2}}}\right)$ and $\bar{z}\left(:=\partial_{t} z^{S^{\rho_{1}, \rho_{2}}}\right)$ satisfy, respectively,

$$
\begin{gathered}
\frac{\partial \widetilde{z}}{\partial t}=\frac{\partial^{2} \widetilde{z}}{\partial x^{2}}+\bar{f}_{\varepsilon, \delta}^{\prime}\left(z^{S^{\rho_{1}, \rho_{2}}}+S^{\rho_{1}, \rho_{2}}\right) \cdot\left(\widetilde{z}+\partial_{x} S^{\rho_{1}, \rho_{2}}\right) ; \\
\widetilde{z}(x, 0)=0, \\
\frac{\partial \bar{z}}{\partial t}=\frac{\partial^{2} \bar{z}}{\partial x^{2}}+\bar{f}_{\varepsilon, \delta}^{\prime}\left(z^{S^{\rho_{1}, \rho_{2}}}+S^{\rho_{1}, \rho_{2}}\right) \cdot\left(\bar{z}+\partial_{t} S^{\rho_{1}, \rho_{2}}\right) ; \\
\bar{z}(x, 0)=0 .
\end{gathered}
$$


Therefore, from Lemmas 3 and 4, we get

$$
\begin{aligned}
\|\tilde{z}\|_{\infty}^{T} & \leq\left\|\partial_{x} S^{\rho_{1}, \rho_{2}}\right\|_{\infty}^{T} \leq C_{\vartheta / 2} C_{\varepsilon, \delta} \cdot \rho_{1}{ }^{\vartheta / 2-1} ; \\
\|\bar{z}\|_{\infty}^{T} & \leq\left\|\partial_{t} S^{\rho_{1}, \rho_{2}}\right\|_{\infty}^{T} \leq C_{\vartheta / 2} C_{\varepsilon, \delta} \cdot \rho_{2}{ }^{9 / 4-1} .
\end{aligned}
$$

In view of (34), (36), (38), and (40), one has

$$
\begin{aligned}
& \left|\bar{u}^{\varepsilon, \delta}(x, t)-\bar{u}^{\varepsilon, \delta}(y, s)\right| \\
& \leq\left|z^{S^{\varepsilon, \delta}}(x, t)-z^{S^{\varepsilon, \delta}}(y, s)\right|+\left|S_{t}^{\varepsilon, \delta}(x)-S_{s}^{\varepsilon, \delta}(y)\right| \\
& \leq\left|z^{S^{\varepsilon, \delta}}(x, t)-z^{S^{\rho_{1}, \rho_{2}}}(x, t)\right| \\
& +\left|z^{S^{\rho_{1}, \rho_{2}}}(x, t)-z^{S^{\rho_{1}, \rho_{2}}}(y, s)\right| \\
& +\left|z^{S^{\rho_{1}, \rho_{2}}}(y, s)-z^{S^{\varepsilon, \delta}}(y, s)\right| \\
& +C_{\varepsilon, \delta}\left(|x-y|^{9 / 2}+|t-s|^{9 / 4}\right) \\
& \leq 2\left\|S^{\varepsilon, \delta}-S^{\rho_{1}, \rho_{2}}\right\|_{\infty}^{T}+\|\tilde{z}\|_{\infty}^{T} \cdot|x-y| \\
& +\|\bar{z}\|_{\infty}^{T} \cdot|t-s|+C_{\varepsilon, \delta}\left(|x-y|^{9 / 2}+|t-s|^{\vartheta / 4}\right) \\
& \leq 2 C_{\vartheta / 2} C_{\varepsilon, \delta}\left(\rho_{1}{ }^{9 / 2}+\rho_{2}{ }^{9 / 4}\right) \\
& +C_{\vartheta / 2} C_{\varepsilon, \delta}\left(\rho_{1}{ }^{\vartheta / 2-1} \cdot|x-y|+\rho_{2}{ }^{\vartheta / 4-1} \cdot|t-s|\right) \\
& +C_{\varepsilon, \delta}\left(|x-y|^{9 / 2}+|t-s|^{\beta / 2}\right) .
\end{aligned}
$$

Set $\rho_{1}:=|x-y|$ and $\rho_{2}:=|t-s| ;$ then

$$
\left|\bar{u}^{\varepsilon, \delta}(x, t)-\bar{u}^{\varepsilon, \delta}(y, s)\right| \leq \gamma_{\varepsilon, \delta}\left(|x-y|^{9 / 2}+|t-s|^{9 / 4}\right),
$$

where $\gamma_{\varepsilon, \delta}:=\left(3 C_{\vartheta / 2}+1\right) C_{\varepsilon, \delta}$ and, for $p \geq 1$, $\sup _{\varepsilon, \delta} \mathbb{E} \gamma_{\varepsilon, \delta}^{p}<\infty$. This yields, for $p \geq 1$,

$$
\begin{aligned}
& \sup _{\varepsilon, \delta} \mathbb{E}\left|\bar{u}^{\varepsilon, \delta}(x, t)-\bar{u}^{\varepsilon, \delta}(y, s)\right|^{p} \\
& \quad \leq C M_{p}\left(|x-y|^{(\vartheta / 2) p}+|t-s|^{(9 / 4) p}\right) .
\end{aligned}
$$

By Lemma 2, we obtain from (43) that

$$
\mathbb{E}|\bar{u}(x, t)-\bar{u}(y, s)|^{p} \leq \widetilde{C}_{p}\left(|x-y|^{(9 / 2) p}+|t-s|^{(9 / 4) p}\right) .
$$

Applying a variant of Garsia’s lemma (see Proposition A.1 and Corollary A.3 in [30]), we conclude that

$$
\begin{aligned}
& |\bar{u}(x, t)-\bar{u}(y, s)| \\
& \quad \leq C_{p}(\omega)\left(|x-y|^{9 / 2-4 / p}+|t-s|^{9 / 4-2 / p}\right) .
\end{aligned}
$$

Since $p$ can be chosen to be arbitrarily large and $\vartheta$ to be as close to 1 as one wants, we have (26).
By Lemma 2.2 of [31], for any $0<\omega<\beta$,

$$
\begin{aligned}
& \left|\int_{S^{1}} G_{t}(x, y) u_{0}(y) d y-\int_{S^{1}} G_{t}(z, y) u_{0}(y) d y\right| \\
& \quad \leq C_{\circlearrowright}\left\|u_{0}\right\|_{\circledast}\left(|t-s|^{\varpi / 2}+|x-z|^{\varpi}\right),
\end{aligned}
$$

and combining (26), we have (28).

The proof is complete.

Denote by $\mathscr{B}\left(C\left(S^{1}\right)\right)$ the $\sigma$-field of all Borel subsets of $C\left(S^{1}\right)$ and by $\mathscr{M}\left(C\left(S^{1}\right)\right)$ the set of all probability measures defined on $\left(C\left(S^{1}\right), \mathscr{B}\left(C\left(S^{1}\right)\right)\right)$. We denote by $u\left(x, t, u_{0}\right)$ the solution of $(1)$ and by $P_{t}\left(u_{0}, \cdot\right)$ the corresponding transition function

$$
P_{t}\left(u_{0}, \Gamma\right)=P\left(u\left(\cdot, t, u_{0}\right) \in \Gamma\right), \quad \Gamma \in \mathscr{B}\left(C\left(S^{1}\right)\right), t>0,
$$

where $u_{0}$ is the initial condition. For $\mu \in \mathscr{M}\left(C\left(S^{1}\right)\right)$, we set

$$
P_{t}^{*} \mu(\Gamma)=\int_{C\left(S^{1}\right)} P_{t}(x, \Gamma) \mu(d x),
$$

where $t \geq 0, \Gamma \in \mathscr{B}\left(C\left(S^{1}\right)\right)$.

Theorem 6. Suppose the hypotheses (H1), (H2), and (F1)-(F3) hold. Then there exists an invariant measure to (1) on $C\left(S^{1}\right)$ for all $\alpha \geq 0, c_{1}>0$, and $c_{2}>0$.

Proof. According to the Krylov-Bogolyubov theorem (see [6]), if there exists $u_{0} \in C\left(S^{1}\right)$ such that the family $P_{t}\left(u_{0}, \cdot\right), t \geq 1$ is uniformly tight, then there exists an invariant measure for (1). We need to show that for any $\varepsilon>0$ there is a compact set $K \subset C\left(S^{1}\right)$ such that

$$
P(u(t) \in K) \geq 1-\varepsilon, \quad \text { for any } t \geq 1,
$$

where $u(t)=u\left(t, u_{0}\right)=u\left(\cdot, t, u_{0}\right)$. On the other hand, for any $t \geq 1$, we have by the Markov property

$$
P(u(t) \in K)=\mathbb{E}\left(P_{1}(u(t-1), K)\right) .
$$

Thus it is enough to show $P(u(1, u(t-1)) \in K) \geq 1-\varepsilon$, for any $t \geq 1$. As $h^{1}(\cdot) \leq u(t-1)(\cdot) \leq h^{2}(\cdot)$, it suffices to find a compact subset $K \subset C\left(S^{1}\right)$ such that

$P_{1}(g, K) \geq 1-\varepsilon, \quad \forall g \in C\left(S^{1}\right)$ with $h^{1} \leq g \leq h^{2}$.

Put

$$
\bar{u}(x, t, g)=u(x, t, g)-\int_{S^{1}} G_{t}(x, y) g(y) d y .
$$

Then $(\bar{u}, \eta, \xi)$ solves a random obstacle problem.

Define

$$
\begin{aligned}
\|f\|_{\beta}=\sup \left\{\frac{|f(x, t)-f(y, s)|}{\left(|x-y|^{2}+(t-s)^{2}\right)^{1 / 2}}:\right. \\
\left.\quad(x, t),(y, s) \in S^{1} \times[0,1],(x, t) \neq(y, s)\right\} .
\end{aligned}
$$


By the Arzela-Ascoli theorem, for all $r>0, K_{r}:=$ $\left\{f ;\|f\|_{\beta} \leq r\right\}$ is a compact subset of $C\left(S^{1} \times[0,1]\right)$. In view of (26), we see that, for given $\varepsilon>0$, there exists $r_{0}$ such that

$$
P\left(\bar{u}(\cdot, \cdot, g) \in K_{r_{0}}^{c}\right) \leq \varepsilon, \quad \forall g \text { with } h^{1} \leq g \leq h^{2} .
$$

Let $K^{\prime}=\left\{\varphi(\cdot, 1): \varphi(\cdot, \cdot) \in K_{r_{0}}\right\}$ which is a compact subset in $C\left(S^{1}\right)$; we obtain $P\left(\bar{u}(\cdot, 1, g) \in K^{\prime}\right) \geq 1-\varepsilon$, for all $g$ with $h^{1} \leq g \leq h^{2}$.

Also, it is easy to see that there is a compact subset $K_{0} \subset$ $C\left(S^{1}\right)$ such that

$$
\left\{\int_{S^{1}} G_{1}(x, y) g(y) d y ; h^{1} \leq g \leq h^{2}\right\} \subset K_{0} .
$$

Define $K=K^{\prime}+K_{0}$. We have

$$
\begin{aligned}
P_{1}(g, K) & =P(u(\cdot, 1, g) \in K) \\
& \geq P\left(\bar{u}(\cdot, 1, g) \in K^{\prime}\right) \geq 1-\varepsilon,
\end{aligned}
$$

for all $g \in C\left(S^{1}\right)$ with $h^{1} \leq g \leq h^{2}$. This finishes the proof.

The following result is the uniqueness of invariant measures.

Theorem 7. Under the assumptions in Theorem 6 and the fact that $|\sigma(\cdot)| \geq L_{0}$ for some constant $L_{0}>0$, there is a unique invariant measure for (1) for all $\alpha \geq 0, c_{1}>0$, and $c_{2}>0$.

Proof of Theorem 7. To prove the uniqueness, we apply coupling method to SPDEs with reflection and singular terms; see in [20] or in [12]. Suppose there are two invariant probabilities $\mu_{1}$ and $\mu_{2}$ which are distributions of initial values $u^{1}(x, 0)$ and $u^{2}(x, 0)$, respectively. And so $u^{1}(x, t)$ and $u^{2}(x, t)$ have these distributions for any $t>0$. Then the total variation distance between $\mu_{1}$ and $\mu_{2}$ satisfies

$$
\operatorname{Var}\left(\mu_{1}-\mu_{2}\right) \leq P\left(\sup _{x \in S^{1}}\left|u^{1}(x, t)-u^{2}(x, t)\right| \neq 0\right) .
$$

Consider the following SPDEs:

$$
\begin{aligned}
\frac{\partial u(x, t)}{\partial t}= & \frac{\partial^{2} u(x, t)}{\partial x^{2}}+f(u(x, t)) \\
& +\sigma(u(x, t)) \dot{W}_{1}(x, t) \\
& +\eta_{1}(x, t)-\xi_{1}(x, t)+\frac{c_{1}}{\left(u(x, t)-h^{1}(x)\right)^{\alpha}} \\
& -\frac{c_{2}}{\left(h^{2}(x)-u(x, t)\right)^{\alpha}}, \\
\frac{\partial v(x, t)}{\partial t}= & \frac{\partial^{2} v(x, t)}{\partial x^{2}}+f(v(x, t)) \\
& +\sigma(v(x, t)) \\
& \times\left[(1-|u(x, t)-v(x, t)| \wedge 1)^{1 / 2} \dot{W}_{1}(x, t)\right. \\
& \left.\quad+(|u(x, t)-v(x, t)| \wedge 1)^{1 / 2} \dot{W}_{2}(x, t)\right]
\end{aligned}
$$

$$
\begin{array}{r}
+\eta_{2}(x, t)-\xi_{2}(x, t)+\frac{c_{1}}{\left(v(x, t)-h^{1}(x)\right)^{\alpha}} \\
-\frac{c_{2}}{\left(h^{2}(x)-v(x, t)\right)^{\alpha}}, \\
u(x, 0)=u^{1}(x, 0), \quad v(x, 0)=u^{2}(x, 0),
\end{array}
$$

where $W_{1}(x, t), W_{2}(x, t)$ are two independent space-time white noises defined on the same probability space $(\Omega, \mathscr{F}, P)$. The existence of solutions of (58) can be obtained by a similar method as that in the paper [11]. Indeed, $(u, v)$ is the limit of the following SPDEs with Lipschitz diffusion coefficients:

$$
\begin{aligned}
\frac{\partial u(x, t)}{\partial t}= & \frac{\partial^{2} u(x, t)}{\partial x^{2}}+f(u(x, t)) \\
& +\sigma(u(x, t)) \dot{W}_{1}(x, t) \\
& +\eta_{1}(x, t)-\xi_{1}(x, t)+\frac{c_{1}}{\left(u(x, t)-h^{1}(x)\right)^{\alpha}} \\
& -\frac{c_{2}}{\left(h^{2}(x)-u(x, t)\right)^{\alpha}}, \\
\frac{\partial v^{n}(x, t)}{\partial t}= & \frac{\partial^{2} v^{n}(x, t)}{\partial x^{2}}+f\left(v^{n}(x, t)\right) \\
& +\sigma\left(v^{n}(x, t)\right) \\
& \times\left[g_{n}\left(\left|u(x, t)-v^{n}(x, t)\right| \wedge 1\right) \dot{W}_{1}(x, t)\right. \\
& \left.+f_{n}\left(\left|u(x, t)-v^{n}(x, t)\right| \wedge 1\right) \dot{W}_{2}(x, t)\right] \\
& +\bar{\eta}_{2}^{n}(x, t)-\bar{\xi}_{2}^{n}(x, t)+\frac{c_{1}}{\left(v^{n}(x, t)-h^{1}(x)\right)^{\alpha}} \\
& -\frac{c_{2}}{\left(h^{2}(x)-v^{n}(x, t)\right)^{\alpha},} \\
u(x, 0)= & u^{1}(x, 0), \quad v(x, 0)=u^{2}(x, 0),
\end{aligned}
$$

where $f_{n}(\cdot)=(\cdot+1 / n)^{1 / 2}-(1 / n)^{1 / 2}$ and $g_{n}(\cdot)=\left(1-f_{n}(\cdot)^{2}\right)^{1 / 2}$.

Therefore, in order to get the uniqueness of invariant measure, it is enough to show

$$
\lim _{t \rightarrow \infty} P\left(\sup _{x \in S^{1}}\left|u^{1}(x, t)-u^{2}(x, t)\right| \neq 0\right)=0,
$$

for given two initial functions $u^{1}(x, 0)$ and $u^{2}(x, 0)$.

Using a similar argument as in the proof of Theorem 2.2 in [12], the details of which we omit, it suffices to establish the following equation when $u^{1}(x, 0) \geq u^{2}(x, 0), x \in S^{1}$ :

$$
\begin{aligned}
& \int_{S^{1}}(u(x, t)-v(x, t)) d x \\
& \quad=\int_{S^{1}}\left(u_{1}(x, 0)-u_{2}(x, 0)\right) d x+C(t)+M(t),
\end{aligned}
$$


where

$$
\begin{aligned}
& M(t)=\int_{0}^{t} \int_{S^{1}} \sigma(u(x, s)) W_{1}(d x, d s) \\
&-\int_{0}^{t} \int_{S^{1}} \sigma(v(x, s)) \\
& \quad \times(1-|u(x, s)-v(x, s)| \wedge 1)^{1 / 2} \\
& \quad \times \dot{W}_{1}(d x, d s) \\
&-\int_{0}^{t} \int_{S^{1}} \sigma(v(x, s))(|u(x, s)-v(x, s)| \wedge 1)^{1 / 2} \\
& \quad \times \dot{W}_{2}(d x, d s),
\end{aligned}
$$

and $C(t)$ is a continuous, adapted, and nonincreasing process.

In view of the reflection and singular terms, we will construct approximating solutions of uniform convergence limits. Consider the following approximating SPDEs:

$$
\begin{aligned}
\frac{\partial u^{\varepsilon, \delta}}{\partial t}= & \frac{\partial^{2} u^{\varepsilon, \delta}}{\partial x^{2}}+f\left(u^{\varepsilon, \delta}\right)+f_{\varepsilon, \delta}\left(u^{\varepsilon, \delta}\right)+\sigma\left(u^{\varepsilon, \delta}\right) \dot{W}_{1} ; \\
\frac{\partial v^{n, \varepsilon, \delta}}{\partial t}= & \frac{\partial^{2} v^{n, \varepsilon, \delta}}{\partial x^{2}}+f\left(v^{n, \varepsilon, \delta}\right)+f_{\varepsilon, \delta}\left(v^{n, \varepsilon, \delta}\right) \\
& +\sigma\left(v^{n, \varepsilon, \delta}\right) \\
& \times\left[g_{n}\left(\left|u^{\varepsilon, \delta}-v^{n, \varepsilon, \delta}\right| \wedge 1\right) \dot{W}_{1}(x, t)\right. \\
& \left.+f_{n}\left(\left|u^{\varepsilon, \delta}-v^{n, \varepsilon, \delta}\right| \wedge 1\right) \dot{W}_{2}(x, t)\right] ; \\
u^{\varepsilon, \delta}(x, 0)= & u^{1}(x, 0), \quad v^{n, \varepsilon, \delta}(x, 0)=u^{2}(x, 0),
\end{aligned}
$$

where $f_{\varepsilon, \delta}$ is the same as in (7).

When $u^{1}(x, 0) \geq u^{2}(x, 0), x \in S^{1}$, we have $u^{\varepsilon, \delta} \geq v^{n, \varepsilon, \delta}$ which can be shown as Lemma 3.1 in [20]. Also, we will assume that $f(u)$ is nonincreasing. Otherwise, consider $\widetilde{u}:=$ $e^{-L t} u$ and $\widetilde{v}:=e^{-L t} v$, where $L$ is the Lipschitz constant in (F1). Then the new drift term $e^{-L t} f\left(e^{L t} x\right)-L x$ is nonincreasing. Thus,

$$
\begin{aligned}
C^{n, \varepsilon, \delta}(t)=\int_{S^{1}}\{ & f\left(u^{\varepsilon, \delta}\right)-f\left(v^{n, \varepsilon, \delta}\right) \\
+ & {\left.\left[f_{\varepsilon, \delta}\left(u^{\varepsilon, \delta}\right)-f_{\varepsilon, \delta}\left(v^{n, \varepsilon, \delta}\right)\right]\right\} d x \leq 0 . }
\end{aligned}
$$

Now we only need $v^{n, \varepsilon, \delta}(x, t) \rightarrow v^{n}(x, t)$ as $\varepsilon, \delta \rightarrow 0$ which will be shown in the lemma below. Letting $\varepsilon, \delta \rightarrow 0$ in the relation of $\int_{S^{1}}\left(u^{\varepsilon, \delta}(x, t)-v^{n, \varepsilon, \delta}(x, t)\right) d x$ from (63) and then sending $n$ to $\infty$, we obtain (61). The proof is complete.

Lemma 8. $v^{n, \varepsilon, \delta}(x, t) \rightarrow v^{n}(x, t)$ in probability on $S^{1} \times[0, T]$, for any $T>0$, as $\varepsilon, \delta \rightarrow 0$.
Proof. Consider the following SPDE:

$$
\begin{aligned}
\frac{\partial \widetilde{v}^{n, \varepsilon, \delta}}{\partial t}= & \frac{\partial^{2} \widetilde{v}^{n, \varepsilon, \delta}}{\partial x^{2}}+f\left(\widetilde{v}^{n, \varepsilon, \delta}\right)+f_{\varepsilon, \delta}\left(\widetilde{v}^{n, \varepsilon, \delta}\right) \\
& +\sigma\left(\widetilde{v}^{n, \varepsilon, \delta}\right) \\
& \times\left[g_{n}\left(\left|u-\widetilde{v}^{n, \varepsilon, \delta}\right| \wedge 1\right) \dot{W}_{1}(x, t)\right. \\
& \left.\quad+f_{n}\left(\left|u-\widetilde{v}^{n, \varepsilon, \delta}\right| \wedge 1\right) \dot{W}_{2}(x, t)\right] ; \\
& \widetilde{v}^{n, \varepsilon, \delta}(x, 0)=u^{2}(x, 0) .
\end{aligned}
$$

Denote the solution by $\widetilde{v}^{n, \varepsilon, \delta}(x, t)$. Let $\widetilde{v}^{n, \varepsilon, \delta}=\widetilde{z}^{n, \varepsilon, \delta}+\widetilde{w}^{n, \varepsilon, \delta}$; here $\widetilde{w}^{n, \varepsilon, \delta}(x, t)$ and $\widetilde{z}^{n, \varepsilon, \delta}(x, t)$ satisfy, respectively,

$$
\begin{gathered}
\frac{\partial \widetilde{w}^{n, \varepsilon, \delta}}{\partial t}=\frac{\partial^{2} \widetilde{w}^{n, \varepsilon, \delta}}{\partial x^{2}}+f\left(v^{n, \varepsilon, \delta}\right)+\sigma\left(v^{n, \varepsilon, \delta}\right) \\
\times\left[g_{n}\left(\left|u-\widetilde{v}^{n, \varepsilon, \delta}\right| \wedge 1\right) \dot{W}_{1}(x, t)\right. \\
\left.\quad+f_{n}\left(\left|u-\widetilde{v}^{n, \varepsilon, \delta}\right| \wedge 1\right) \dot{W}_{2}(x, t)\right] ; \\
\widetilde{w}^{n, \varepsilon, \delta}(x, 0)=u^{2}(x, 0), \\
\frac{\partial \widetilde{z}^{n, \varepsilon, \delta}}{\partial t}=\frac{\partial^{2} \widetilde{z}^{n, \varepsilon, \delta}}{\partial x^{2}}+f_{\varepsilon, \delta}\left(\widetilde{z}^{n, \varepsilon, \delta}+\widetilde{w}^{n, \varepsilon, \delta}\right) ; \\
\widetilde{z}^{n, \varepsilon, \delta}(x, 0)=0 .
\end{gathered}
$$

Similarly, define $w^{n, \varepsilon, \delta}(x, t)$ and $z^{n, \varepsilon, \delta}(x, t)$ satisfy, respectively,

$$
\begin{gathered}
\frac{\partial w^{n, \varepsilon, \delta}}{\partial t}=\frac{\partial^{2} w^{n, \varepsilon, \delta}}{\partial x^{2}}+f\left(v^{n, \varepsilon, \delta}\right)+\sigma\left(v^{n, \varepsilon, \delta}\right) \\
\times\left[g_{n}\left(\left|u^{\varepsilon, \delta}-v^{n, \varepsilon, \delta}\right| \wedge 1\right) \dot{W}_{1}(x, t)\right. \\
\left.+f_{n}\left(\left|u^{\varepsilon, \delta}-v^{n, \varepsilon, \delta}\right| \wedge 1\right) \dot{W}_{2}(x, t)\right] ; \\
w^{n, \varepsilon, \delta}(x, 0)=u^{2}(x, 0), \\
\frac{\partial z^{n, \varepsilon, \delta}}{\partial t}=\frac{\partial^{2} z^{n, \varepsilon, \delta}}{\partial x^{2}}+f_{\varepsilon, \delta}\left(z^{n, \varepsilon, \delta}+w^{n, \varepsilon, \delta}\right) ; \\
z^{n, \varepsilon, \delta}(x, 0)=0 .
\end{gathered}
$$

Then $v^{n, \varepsilon, \delta}=z^{n, \varepsilon, \delta}+w^{n, \varepsilon, \delta}$.

From similar proof in Proposition 3.1 in [11], one has

$$
\left\|z^{n, \varepsilon, \delta}-\widetilde{z}^{n, \varepsilon, \delta}\right\|_{\infty}^{T} \leq\left\|w^{n, \varepsilon, \delta}-\widetilde{w}^{n, \varepsilon, \delta}\right\|_{\infty}^{T} .
$$

Thus,

$$
\left\|v^{n, \varepsilon, \delta}-\widetilde{v}^{n, \varepsilon, \delta}\right\|_{\infty}^{T} \leq 2\left\|w^{n, \varepsilon, \delta}-\widetilde{w}^{n, \varepsilon, \delta}\right\|_{\infty}^{T} .
$$

Now we will show that $\left\|v^{n, \varepsilon, \delta}-\widetilde{v}^{n, \varepsilon, \delta}\right\|_{\infty}^{T} \rightarrow 0$ in probability as $\mathcal{\varepsilon}, \delta \rightarrow 0$. 
As the similar proof in Donati-Martin and Pardoux [2], it can be shown that $\sup _{\varepsilon, \delta} \mathbb{E}\left(\left\|v^{n, \varepsilon, \delta}\right\|_{\infty}^{T}\right)^{p}<\infty$, for arbitrarily large $p$ and any $T>0$. Set the stopping time $\tau_{M}:=$ $\inf \left\{t ; \sup _{x \in S^{1}}\left|v^{n, \varepsilon, \delta}(x, t)\right|>M\right\}$. For fixed $M>0$, we have

$$
\begin{gathered}
\mathbb{E}\left(\left\|w^{n, \varepsilon, \delta}\left(t \wedge \tau_{M}\right)-\widetilde{w}^{n, \varepsilon, \delta}\left(t \wedge \tau_{M}\right)\right\|_{\infty}^{T}\right)^{2} \\
\leq C_{1} \int_{0}^{T} \mathbb{E}\left(\left\|f\left(v^{n, \varepsilon, \delta}\right)-f\left(\widetilde{v}^{n, \varepsilon, \delta}\right)\right\|_{\infty}^{t \wedge \tau_{M}}\right)^{2} d s \\
+C_{2} \int_{0}^{T} \mathbb{E}\left(\| \sigma\left(v^{n, \varepsilon, \delta}\right) g_{n}\left(\left|u^{\varepsilon, \delta}-v^{n, \varepsilon, \delta}\right| \wedge 1\right)\right. \\
-\sigma\left(\widetilde{v}^{n, \varepsilon, \delta}\right) \\
\left.\times g_{n}\left(\left|u-\widetilde{v}^{n, \varepsilon, \delta}\right| \wedge 1\right) \|_{\infty}^{t \wedge \tau_{M}}\right)^{2} d s \\
+C_{2} \int_{0}^{T} \mathbb{E}\left(\| \sigma\left(v^{n, \varepsilon, \delta}\right) f_{n}\left(\left|u^{\varepsilon, \delta}-v^{n, \varepsilon, \delta}\right| \wedge 1\right)\right. \\
-\sigma\left(\widetilde{v}^{n, \varepsilon, \delta}\right) \\
\left.\times f_{n}\left(\left|u-\widetilde{v}^{n, \varepsilon, \delta}\right| \wedge 1\right) \|_{\infty}^{t \wedge \tau_{M}}\right)^{2} d s \\
\leq C_{1} \int_{0}^{T} \mathbb{E}\left(\left\|w^{n, \varepsilon, \delta}-\widetilde{w}^{n, \varepsilon, \delta}\right\|_{\infty}^{t \wedge \tau_{M}}\right)^{2} d s \\
+C_{2} \int_{0}^{T} \mathbb{E}\left(\left\|\sigma\left(v^{n, \varepsilon, \delta}\right)\right\|_{\infty}^{t \wedge \tau_{M}}\right. \\
+C_{1} \epsilon+C_{2} \int_{0}^{T} \mathbb{E}\left(\left\|w^{n, \varepsilon, \delta}-\widetilde{w}^{n, \varepsilon, \delta}\right\|_{\infty}^{t \wedge \tau_{M}}\right)^{2} d s, \\
+u^{\varepsilon, \delta}-u \|_{\infty}^{t \wedge \tau_{M}} \\
\left.\left.+w^{n, \varepsilon, \delta}-\widetilde{w}^{n, \varepsilon, \delta} \|_{\infty}^{t \wedge \tau_{M}}\right]\right)^{2} d s
\end{gathered}
$$

where the constants change in each line and $\epsilon \rightarrow 0$ as $\varepsilon, \delta \rightarrow 0$ because of Lemma 2. By the Gronwall inequality, we get $\mathbb{E}\left(\left\|w^{n, \varepsilon, \delta}\left(t \wedge \tau_{M}\right)-\widetilde{w}^{n, \varepsilon, \delta}\left(t \wedge \tau_{M}\right)\right\|_{\infty}^{T}\right)^{2} \rightarrow 0$ as $\varepsilon, \delta \rightarrow$ 0 . Hence, $\mathbb{E}\left(\left\|v^{n, \varepsilon, \delta}\left(t \wedge \tau_{M}\right)-\widetilde{v}^{n, \varepsilon, \delta}\left(t \wedge \tau_{M}\right)\right\|_{\infty}^{T}\right)^{2} \rightarrow 0$. So $\left\|v^{n, \varepsilon, \delta}-\widetilde{v}^{n, \varepsilon, \delta}\right\|_{\infty}^{T} \rightarrow 0$ in probability. In fact, for every $\epsilon_{0}>0$, set the event $A^{n, \varepsilon, \delta}:=\left\{\left\|v^{n, \varepsilon, \delta}-\widetilde{v}^{n, \varepsilon, \delta}\right\|_{\infty}^{T}>\epsilon_{0}\right\}$,

$$
\begin{aligned}
P\left(A^{n, \varepsilon, \delta}\right)= & P\left(A^{n, \varepsilon, \delta}, \tau_{M} \leq T\right)+P\left(A^{n, \varepsilon, \delta}, \tau_{M}>T\right) \\
\leq & P\left(\tau_{M} \leq T\right) \\
& +\frac{\mathbb{E}\left(\left\|w^{n, \varepsilon, \delta}\left(t \wedge \tau_{M}\right)-\widetilde{w}^{n, \varepsilon, \delta}\left(t \wedge \tau_{M}\right)\right\|_{\infty}^{T}\right)^{2}}{\epsilon_{0}{ }^{2}}
\end{aligned}
$$

$$
\begin{aligned}
& \leq \frac{\mathbb{E}\left(\left\|v^{n, \varepsilon, \delta}\right\|_{\infty}^{T}\right)^{2}}{M^{2}} \\
&+\frac{\mathbb{E}\left(\left\|w^{n, \varepsilon, \delta}\left(t \wedge \tau_{M}\right)-\widetilde{w}^{n, \varepsilon, \delta}\left(t \wedge \tau_{M}\right)\right\|_{\infty}^{T}\right)^{2}}{\epsilon_{0}{ }^{2}} \\
& \longrightarrow 0, \quad \text { as } \varepsilon, \delta \longrightarrow 0, M \longrightarrow \infty .
\end{aligned}
$$

Since we can apply Lemma 2 to conclude that $u^{\varepsilon, \delta}(x, t) \rightarrow$ $u(x, t), \widetilde{v}^{n, \varepsilon, \delta}(x, t) \rightarrow v^{n}(x, t)$ uniformly on $S^{1} \times[0, T]$ as $\varepsilon, \delta \rightarrow 0$. Therefore, $v^{n, \varepsilon, \delta}(x, t) \rightarrow v^{n}(x, t)$ in probability on $S^{1} \times[0, T]$ as $\varepsilon, \delta \rightarrow 0$.

\section{Strong Feller Property}

In this section, we consider the strong Feller property of the solution of (1). Let $H=L^{2}\left(S^{1}\right)$. If $\varphi \in B_{b}(H)$ (the Banach space of all real bounded Borel functions, endowed with the sup norm), we define, for $x \in S^{1}, 0 \leq t \leq T$, and $g \in H$, $P_{t} \varphi(g)=\mathbb{E} \varphi(u(x, t, g))$.

Theorem 9. Under the hypotheses (H1), (H2), and (F1)-(F3) and the fact that $p_{1} \leq|\sigma(\cdot)| \leq p_{2}$, for some constants $p_{1}, p_{2}>$ 0 , for any $T>0$, there exists a constant $C_{T}^{\prime}$ such that, for all $\varphi \in B_{b}(H)$ and $t \in(0, T]$,

$$
\left|P_{t} \varphi\left(u_{0}^{1}\right)-P_{t} \varphi\left(u_{0}^{2}\right)\right| \leq \frac{C_{T}^{\prime}}{\sqrt{t}}\|\varphi\|_{\infty}\left|u_{0}^{1}-u_{0}^{2}\right|_{H}
$$

for $u_{0}^{1}, u_{0}^{2} \in H$ with $h^{1}(x) \leq u_{0}^{1}(x), u_{0}^{2}(x) \leq h^{2}(x)$, where $\|\varphi\|_{\infty}=\sup _{u_{0} \in H}\left|\varphi\left(u_{0}\right)\right|$. In particular, $P_{t}, t>0$ is strong Feller.

Proof. Choose a nonnegative function $\phi \in C_{0}^{\infty}(R)$ with $\int_{R} \phi(x)=1$ and denote

$$
\begin{aligned}
& f_{n}(\zeta):=n \int_{R} \phi(n(\zeta-y)) f(y) d y, \\
& \sigma_{n}(\zeta):=n \int_{R} \phi(n(\zeta-y)) \sigma(y) d y, \\
& k_{n, \varepsilon, \delta}(\zeta, x):=n \int_{R} \phi(n(\zeta-y)) \\
& \times\left[\frac{1}{\left[\delta+\left[\left(y-h^{1}(x)\right) \vee 0\right]^{2}\right]^{\alpha / 2}}\right. \\
& \left.\quad-\frac{1}{\left[\varepsilon+\left[\left(h^{2}(x)-y\right) \vee 0\right]^{2}\right]^{\alpha / 2}}\right] d y,
\end{aligned}
$$




$$
\begin{aligned}
l_{n, \varepsilon, \delta}(\zeta, x):= & k_{n, \varepsilon, \delta}(\zeta, x) \\
& +\left(\frac{\arctan \left(\left[\left(\zeta-h^{1}(x)\right) \wedge 0\right]^{2}\right)}{\delta}\right. \\
& \left.-\frac{\arctan \left(\left[\left(h^{2}(x)-\zeta\right) \wedge 0\right]^{2}\right)}{\varepsilon}\right) .
\end{aligned}
$$

$$
\begin{aligned}
& +\int_{0}^{t} \int_{S^{1}} G_{t-s}(x, y) \sigma_{n}^{\prime}\left(u_{n}^{\varepsilon, \delta}\left(y, s, u_{0}\right)\right) \\
& \times X_{n}^{\varepsilon, \delta}(y, s) W(d y, d s) \\
& +\int_{0}^{t} \int_{S^{1}} G_{t-s}(x, y) \frac{\partial}{\partial \zeta} l_{n, \varepsilon, \delta}\left(u_{n}^{\varepsilon, \delta}\left(y, s, u_{0}\right), y\right) \\
& \times X_{n}^{\varepsilon, \delta}(y, s) d y d s .
\end{aligned}
$$

So $f_{n}, \sigma_{n}, l_{n, \varepsilon, \delta}$ are smooth with respect to $\zeta$ and $f_{n}(\zeta) \rightarrow$ $f(\zeta), \sigma_{n}(\zeta) \rightarrow \sigma(\zeta)$, and $l_{n, \varepsilon, \delta}(\zeta, x) \rightarrow f_{\varepsilon, \delta}(\zeta)$ as $n \rightarrow \infty$.

Let

$$
\begin{aligned}
& u_{n}^{\varepsilon, \delta}\left(x, t, u_{0}\right) \\
& =\int_{S^{1}} G_{t}(x, y) u_{0}(y) d y \\
& \quad+\int_{0}^{t} \int_{S^{1}} G_{t-s}(x, y) f_{n}\left(u_{n}^{\varepsilon, \delta}\left(y, s, u_{0}\right)\right) d y d s \\
& \quad+\int_{0}^{t} \int_{S^{1}} G_{t-s}(x, y) \sigma_{n}\left(u_{n}^{\varepsilon, \delta}\left(y, s, u_{0}\right)\right) W(d y, d s) \\
& \quad+\int_{0}^{t} \int_{S^{1}} G_{t-s}(x, y) l_{n, \varepsilon, \delta}\left(u_{n}^{\varepsilon, \delta}\left(y, s, u_{0}\right), y\right) d y d s .
\end{aligned}
$$

Using similar arguments as in [23], we can show that, for any fixed $\varepsilon, \delta$ and $p \geq 1$,

$$
\lim _{n \rightarrow \infty} \sup _{t \in[0, T]} \mathbb{E}\left(\left|u_{n}^{\varepsilon, \delta}\left(t, \cdot, u_{0}\right)-u^{\varepsilon, \delta}\left(t, \cdot, u_{0}\right)\right|_{H}^{p}\right)=0
$$

Furthermore, in view of Lemma 7.1.5 in [6] and Lemma 2, it is enough to prove that there exists a constant $C_{T}^{\prime}$, independent of $\varepsilon, \delta$ and $n$, such that

$$
\left|P_{t}^{n, \varepsilon, \delta} \varphi\left(u_{0}^{1}\right)-P_{t}^{n, \varepsilon, \delta} \varphi\left(u_{0}^{2}\right)\right| \leq \frac{C_{T}^{\prime}}{\sqrt{t}}\|\varphi\|_{\infty}\left|u_{0}^{1}-u_{0}^{2}\right|_{H}
$$

where $P_{t}^{n, \varepsilon, \delta} \varphi\left(u_{0}\right):=\mathbb{E}\left(\varphi\left(u_{n}^{\varepsilon, \delta}\left(\cdot, \cdot, u_{0}\right)\right)\right)$ and $u_{0}^{1}, u_{0}^{2} \in H$.

From Theorem 5.4.1 in [6], $u_{n}^{\varepsilon, \delta}\left(\cdot, \cdot, u_{0}\right)$ is continuously differentiable with respect to $u_{0}$. Denote by $X_{n}^{\varepsilon, \delta}(x, t):=$ $\left(D u_{n}^{\varepsilon, \delta}\left(\cdot, \cdot, u_{0}\right)\left(\bar{u}_{0}\right)\right)(x, t)$ the directional derivative of $u_{n}^{\varepsilon, \delta}\left(\cdot, \cdot, u_{0}\right)$ at $u_{0}$ in the direction of $\bar{u}_{0}$ and it satisfies the mild form of a SPDE as follows:

$$
\begin{aligned}
X_{n}^{\varepsilon, \delta}(x, t)= & \int_{S^{1}} G_{t}(x, y) \bar{u}_{0}(y) d y \\
& +\int_{0}^{t} \int_{S^{1}} G_{t-s}(x, y) f_{n}^{\prime}\left(u_{n}^{\varepsilon, \delta}\left(y, s, u_{0}\right)\right) \\
& \times X_{n}^{\varepsilon, \delta}(y, s) d y d s
\end{aligned}
$$

Since $(\partial / \partial \zeta) l_{n, \varepsilon, \delta}\left(u_{n}^{\varepsilon, \delta}\left(y, s, u_{0}\right), y\right) \leq 0$, we use similar arguments as that in [23] to get

$$
\sup _{\varepsilon, \delta \geq 0, t \in[0, T]} \mathbb{E}\left(\int_{S^{1}}\left(X_{n}^{\varepsilon, \delta}(y, t)\right)^{2} d y\right) \leq C\left|\bar{u}_{0}\right|_{H^{\prime}}^{2}
$$

where $C$ is a constant. By Elworthy-Li formula (Lemma 7.1.3 in [6]), we obtain

$$
\left|\left\langle D P_{t} \varphi\left(u_{0}\right), \bar{u}_{0}\right\rangle\right|^{2} \leq \frac{C}{p_{1}^{2} t}\|\varphi\|_{\infty}^{2}\left|\bar{u}_{0}\right|_{H}^{2}
$$

This implies inequality (77) which completes the proof.

\section{Large Deviation Principle}

We consider a small perturbation of the equation of (1); that is,

$$
\begin{gathered}
\frac{\partial u^{\epsilon}}{\partial t}=\frac{\partial^{2} u^{\epsilon}}{\partial x^{2}}+f\left(u^{\epsilon}\right)+\frac{c_{1}}{\left(u^{\epsilon}-h^{1}\right)^{\alpha}}-\frac{c_{2}}{\left(h^{2}-u^{\epsilon}\right)^{\alpha}} \\
+\epsilon \sigma(u) \dot{W}+\eta^{\epsilon}-\xi^{\epsilon} ; \\
u^{\epsilon}(x, 0)=u_{0}(x) .
\end{gathered}
$$

As a similar discussion in [11], for any $\epsilon>0$, there exists a continuous solution $u^{\epsilon}$ satisfying

$$
\begin{aligned}
u^{\epsilon}(x, t)= & \int_{S^{1}} G_{t}(x, y) u_{0}(y) d y \\
& +\int_{0}^{t} \int_{S^{1}} G_{t-s}(x, y) \\
& \times\left(f\left(u^{\epsilon}(y, s)\right)+\frac{c_{1}}{\left(u^{\epsilon}(y, s)-h^{1}(y)\right)^{\alpha}}\right. \\
& +\epsilon \int_{0}^{t} \int_{S^{1}} G_{t-s}(x, y) \sigma\left(u^{\epsilon}(y, s)\right) W(d y, d s) \\
& +\int_{0}^{t} \int_{S^{1}} G_{t-s}(x, y)\left(\eta^{\epsilon}(d y d s)-\xi^{\epsilon}(d y d s)\right) .
\end{aligned}
$$


The Cameron-Martin space associated with the white noise $\left\{W(x, t),(x, t) \in S^{1} \times[0, T]\right\}$ is given by

$$
\begin{gathered}
\mathscr{H}=\left\{h(x, t)=\int_{0}^{x} \int_{0}^{t} \dot{h}(y, s) d y d s ;\right. \\
\left.\int_{S^{1}} \int_{0}^{T}(\dot{h}(y, s))^{2} d y d s<\infty\right\},
\end{gathered}
$$

endowed with norm $\|h\|_{\mathscr{H}}=\left(\int_{S^{1}} \int_{0}^{T}(\dot{h}(y, s))^{2} d y d s\right)^{1 / 2}$.

Consider the following PDE (the skeleton equation):

$$
\begin{gathered}
\frac{\partial u^{h}}{\partial t}=\frac{\partial^{2} u^{h}}{\partial x^{2}}+f\left(u^{h}\right)+\frac{c_{1}}{\left(u^{h}-h^{1}\right)^{\alpha}}-\frac{c_{2}}{\left(h^{2}-u^{h}\right)^{\alpha}} \\
+\sigma\left(u^{h}\right) \dot{h}+\eta^{h}-\xi^{h} ; \\
u^{h}(x, 0)=u_{0}(x) ; \\
h^{1}(x) \leq u^{h}(x, t) \leq h^{2}(x) .
\end{gathered}
$$

As the analogous discussion in [11], for any $h \in \mathscr{H}$, there exists a continuous solution $u^{h}$ satisfying

$$
\begin{aligned}
u^{h}(x, t)= & \int_{S^{1}} G_{t}(x, y) u_{0}(y) d y \\
& +\int_{0}^{t} \int_{S^{1}} G_{t-s}(x, y) \\
& \times\left(f\left(u^{h}(y, s)\right)+\frac{c_{1}}{\left(u^{h}(y, s)-h^{1}(y)\right)^{\alpha}}\right. \\
& +\int_{0}^{t} \int_{S^{1}} G_{t-s}(x, y) \sigma\left(u^{h}(y, s)\right) \dot{h}(y, s) d y d s \\
& +\int_{0}^{t} \int_{S^{1}} G_{t-s}(x, y)\left(h^{h}(d y d s)-\xi^{h}(d y d s)\right) .
\end{aligned}
$$

We state our main result in this section.

Theorem 10. The laws $\mu^{\epsilon}$ of the $\left\{u^{\epsilon}\right\}_{\epsilon>0}$ satisfy a large deviation principle on $D:=\left\{u(x, t) \in C\left(S^{1} \times[0, T]\right) ; h^{1}(x) \leq u(x, t) \leq\right.$ $\left.h^{2}(x)\right\}$ with the rate function

$$
I(f)=\inf \left\{\frac{1}{2}\|h\|_{\mathscr{H}}^{2} ; u^{h}=f\right\},
$$

with the convention $\inf \{\emptyset\}=\infty$; that is,

(i) for any closed subset $A \subset D$,

$$
\limsup _{\epsilon \rightarrow 0} \epsilon^{2} \log \mu^{\epsilon}(A) \leq-\inf _{f \in A} I(f) ;
$$

(ii) for any open set $B \subset D$,

$$
\liminf _{\epsilon \rightarrow 0} \epsilon^{2} \log \mu^{\epsilon}(B) \geq-\inf _{f \in B} I(f) .
$$

Proof. In order to prove Theorem 10, by Theorem 4.4 in [27], it suffices to check the following.

(i) For any $a>0, u^{h}$ is a continuous mapping from $\{h$ : $\left.\|h\|_{\mathscr{H}} \leq a\right\}$ into $C\left(S^{1} \times[0, T]\right)$.

(ii) For a family $\left\{h^{\epsilon}\right\} \subset A_{N}$, where $A_{N}:=\{h \in$ $\mathscr{H}, ; h(x, t)$ is $\mathscr{F}_{t}-$ measurable, $\|h\|_{\mathscr{H}} \leq N P-$ a.s.\}, $0<N<\infty$, that converges in distribution to $h$, $u^{\epsilon, h^{\epsilon}}$ converges in distribution to $u^{h}$, where $u^{\epsilon, h^{\epsilon}}$ solves the following SPDE:

$$
\begin{aligned}
\frac{\partial u^{\epsilon, h^{\epsilon}}}{\partial t}= & \frac{\partial^{2} u^{\epsilon, h^{\epsilon}}}{\partial x^{2}}+f\left(u^{\epsilon, h^{\epsilon}}\right)+\frac{c_{1}}{\left(u^{\epsilon, h^{\epsilon}}-h^{1}\right)^{\alpha}} \\
& -\frac{c_{2}}{\left(h^{2}-u^{\epsilon, h^{\epsilon}}\right)^{\alpha}}+\epsilon \sigma\left(u^{\epsilon, h^{\epsilon}}\right) \dot{W} \\
& +\sigma\left(u^{\epsilon, h^{\epsilon}}\right) \dot{h}^{\epsilon}+\eta^{\epsilon, h^{\epsilon}}-\xi^{\epsilon, h^{\epsilon}} ; \\
& u^{\epsilon, h^{\epsilon}}(x, 0)=u_{0}(x) .
\end{aligned}
$$

Let

$$
\begin{aligned}
v^{h_{i}}(x, t)= & \int_{S^{1}} G_{t}(x, y) u_{0}(y) d y \\
& +\int_{0}^{t} \int_{S^{1}} G_{t-s}(x, y) f\left(u^{h_{i}}(y, s)\right) d y d s \\
& +\int_{0}^{t} \int_{S^{1}} G_{t-s}(x, y) \sigma\left(u^{h_{i}}(y, s)\right) \dot{h}_{\mathrm{i}}(y, s) d y d s
\end{aligned}
$$

with $i=1,2$. In terms of (36), we have

$$
\left\|u^{h_{1}}-u^{h_{2}}\right\|_{\infty}^{T} \leq 2\left\|v^{h_{1}}-v^{h_{2}}\right\|_{\infty}^{T} .
$$

Proof of (i). We need to prove that, for fixed $a>0, h_{1}, h_{2} \in$ $\left\{h \in \mathscr{H}:\|h\|_{\mathscr{H}} \leq a\right\}$,

$$
\left\|u^{h_{1}}-u^{h_{2}}\right\|_{\infty}^{T} \leq C\left\|h_{1}-h_{2}\right\|_{\mathscr{H}}
$$

where $u^{h_{1}}, u^{h_{2}}$ are the solution of (84) associated with $h_{1}, h_{2}$, respectively. Let

$$
\begin{aligned}
v^{h_{i}}(x, t)= & \int_{S^{1}} G_{t}(x, y) u_{0}(y) d y \\
& +\int_{0}^{t} \int_{S^{1}} G_{t-s}(x, y) f\left(u^{h_{i}}(y, s)\right) d y d s \\
& +\int_{0}^{t} \int_{S^{1}} G_{t-s}(x, y) \sigma\left(u^{h_{i}}(y, s)\right) \dot{h}_{i}(y, s) d y d s
\end{aligned}
$$


with $i=1,2$. In terms of (36), we have

$$
\begin{aligned}
& \left\|u^{h_{1}}-u^{h_{2}}\right\|_{\infty}^{T} \\
& \leq 2\left\|v^{h_{1}}-v^{h_{2}}\right\|_{\infty}^{T} \\
& \leq 2 \| \int_{0}^{t} \int_{S^{1}} G_{t-s}(x, y) \\
& \times\left(f\left(u^{h_{1}}(y, s)\right)\right. \\
& +2 \| \int_{0}^{t} \int_{S^{1}} G_{t-s}(x, y) \\
& \times\left(\sigma\left(u^{h_{1}}(y, s)\right) \dot{h}_{1}(y, s)\right. \\
& \left.\quad-\sigma\left(u^{h_{2}}(y, s)\right)\right) d y d s \|_{\infty}^{T} \\
& \left.\quad=I_{1}+I_{2} . \quad \dot{h}_{2}(y, s)\right) d y d s \|_{\infty}^{T}
\end{aligned}
$$

We know

$$
\begin{aligned}
& I_{1} \leq C\left(\int_{0}^{T}\left(\left\|u^{h_{1}}-u^{h_{2}}\right\|_{\infty}^{t}\right)^{2} d t\right)^{1 / 2}, \\
& I_{2} \leq C \|\left(\int_{0}^{t} \int_{S^{1}}\left|G_{t-s}(x, y)\right|^{2} d y d s\right)^{1 / 2} \\
& \times\left(\int_{0}^{t} \int_{S^{1}} \mid \sigma\left(u^{h_{1}}(y, s)\right) \dot{h}_{1}(y, s)\right. \\
&\left.-\left.\sigma\left(u^{h_{2}}(y, s)\right) \dot{h}_{2}(y, s)\right|^{2} d y d s\right)^{1 / 2} \|_{\infty}^{T} .
\end{aligned}
$$$$
\leq C\left\|h_{1}-h_{2}\right\|_{\mathscr{H}} \text {. }
$$

Then using Gronwall's inequality, we obtain

$$
\left\|u^{h_{1}}-u^{h_{2}}\right\|_{\infty}^{T} \leq C\left\|h_{1}-h_{2}\right\|_{\mathscr{H}} \text {. }
$$

Proof of (ii). It is similar to that in [26] with the help of inequality (91).

Thus the proof of Theorem 10 is completed.

\section{Conflict of Interests}

The authors declare that there is no conflict of interests regarding the publication of this paper.

\section{Acknowledgments}

The authors are grateful to the anonymous referees for their valuable comments and suggestions. This work was supported by the Fundamental Research Funds for the Central Universities, no. 2013RC0906, and by NSFC, nos. 11101419 and 11371362 .

\section{References}

[1] D. Nualart and E. Pardoux, "White noise driven quasilinear SPDEs with reflection," Probability Theory and Related Fields, vol. 93, no. 1, pp. 77-89, 1992.

[2] C. Donati-Martin and E. Pardoux, "White noise driven SPDEs with reflection," Probability Theory and Related Fields, vol. 95, no. 1, pp. 1-24, 1993.

[3] T. Funaki and S. Olla, "Fluctuations for $\nabla_{\varphi}$ interface model on a wall," Stochastic Processes and their Applications, vol. 94, no. 1, pp. 1-27, 2001.

[4] J.-D. Deuschel and G. Giacomin, "Entropic repulsion for massless fields," Stochastic Processes and their Applications, vol. 89, no. 2, pp. 333-354, 2000.

[5] J. L. Lebowitz and C. Maes, "The effect of an external field on an interface, entropic repulsion," Journal of Statistical Physics, vol. 46, no. 1-2, pp. 39-49, 1987.

[6] G. da Prato and J. Zabczyk, Stochastic Equations in Infinite Dimensions, Encyclopedia of Mathematics and Its Applications, Cambridge University Press, Cambridge, UK, 1992.

[7] A. Debussche and L. Zambotti, "Conservative stochastic CahnHilliard equation with reflection," The Annals of Probability, vol. 35, no. 5, pp. 1706-1739, 2007.

[8] M. Röckner, F.-Y. Wang, and T. Zhang, "Stochastic generalized porous media equations with reflection," Stochastic Processes and their Applications, vol. 123, no. 11, pp. 3943-3962, 2013.

[9] A. Debussche and L. Goudenège, "Stochastic Cahn-Hilliard equation with double singular nonlinearities and two reflections," SIAM Journal on Mathematical Analysis, vol. 43, no. 3 , pp. 1473-1494, 2011.

[10] Y. Otobe, "Stochastic partial differential equations with two reflecting walls," Journal of Mathematical Sciences, The University of Tokyo, vol. 13, no. 2, pp. 129-144, 2006.

[11] J. Yang and J. L. Zhai, "SPDEs with two reflecting walls," 2014.

[12] J. Yang and T. S. Zhang, "The existence and uniqueness of invariant measure of SPDEs with two reflecting walls," Journal of Theoretical Probability, pp. 1-15, 2012.

[13] T. Zhang and J. Yang, "White noise driven SPDEs with two reflecting walls," Infinite Dimensional Analysis, Quantum Probability and Related Topics, vol. 14, no. 4, pp. 647-659, 2011.

[14] C. Mueller, "Long-time existence for signed solutions of the heat equation with a noise term," Probability Theory and Related Fields, vol. 110, no. 1, pp. 51-68, 1998.

[15] C. Mueller and E. Pardoux, "The critical exponent for a stochastic PDE to hit zero," in Stochastic Analysis, Control, Optimization and Applications, pp. 325-338, Birkhäuser, Boston, Mass, USA, 1999.

[16] L. Zambotti, "A reflected stochastic heat equation as symmetric dynamics with respect to the 3-d Bessel bridge," Journal of Functional Analysis, vol. 180, no. 1, pp. 195-209, 2001.

[17] L. Zambotti, "Integration by parts on $\delta$-bessel bridges, $\delta>3$ and related SPDEs," The Annals of Probability, vol. 31, no. 1, pp. 323-348, 2003

[18] R. C. Dalang, C. Mueller, and L. Zambotti, "Hitting properties of parabolic SPDE'S with reflection," The Annals of Probability, vol. 34, no. 4, pp. 1423-1450, 2006.

[19] R. Sowers, "Large deviations for the invariant measure of a reaction-diffusion equation with non-Gaussian perturbations," Probability Theory and Related Fields, vol. 92, no. 3, pp. 393-421, 1992. 
[20] C. Mueller, "Coupling and invariant measures for the heat equation with noise," The Annals of Probability, vol. 21, no. 4, pp. 2189-2199, 1993.

[21] S. Peszat and J. Zabczyk, "Strong Feller property and irreducibility for diffusions on Hilbert spaces," The Annals of Probability, vol. 23, no. 1, pp. 157-172, 1995.

[22] Y. Otobe, "Invariant measures for SPDEs with reflection," Journal of Mathematical Sciences, The University of Tokyo, vol. 11, no. 4, pp. 425-446, 2004.

[23] T. Zhang, "White noise driven SPDEs with reflection: strong Feller properties and Harnack inequalities," Potential Analysis, vol. 33, no. 2, pp. 137-151, 2010.

[24] A. Dembo and O. Zeitouni, Large Deviations Techniques and Applications, Jones and Bartlett, Boston, Mass, USA, 1993.

[25] S. Cerrai and M. Röckner, "Large deviations for stochastic reaction-diffusion systems with multiplicative noise and nonLipschitz reaction term," The Annals of Probability, vol. 32, no. 1B, pp. 1100-1139, 2004.

[26] T. Xu and T. Zhang, "White noise driven SPDEs with reflection: existence, uniqueness and large deviation principles," Stochastic Processes and their Applications, vol. 119, no. 10, pp. 3453-3470, 2009.

[27] A. Budhiraja and P. Dupuis, "A variational representation for positive functionals of infinite dimensional Brownian motion," Probability and Mathematical Statistics, vol. 20, no. 1, pp. 39-61, 2000.

[28] P. Dupuis and R. S. Ellis, A Weak Convergence Approach to the Theory of Large Deviations, John Wiley \& Sons, New York, NY, USA, 1997.

[29] R. C. Dalang and T. S. Zhang, "Hölder continuity of solutions of SPDEs with reflection," Communications in Mathematics and Statistics, vol. 1, no. 2, pp. 133-142, 2013.

[30] R. C. Dalang, D. Khoshnevisan, and E. Nualart, "Hitting probabilities for systems of non-linear stochastic heat equations with additive noise," ALEA: Latin American Journal of Probability and Mathematical Statistics, vol. 3, pp. 231-271, 2007.

[31] F. Chenal and A. Millet, "Uniform large deviations for parabolic SPDEs and applications," Stochastic Processes and their Applications, vol. 72, no. 2, pp. 161-186, 1997. 


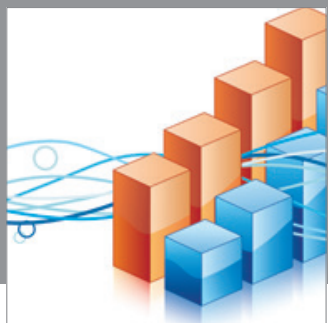

Advances in

Operations Research

mansans

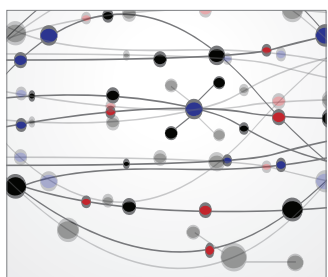

The Scientific World Journal
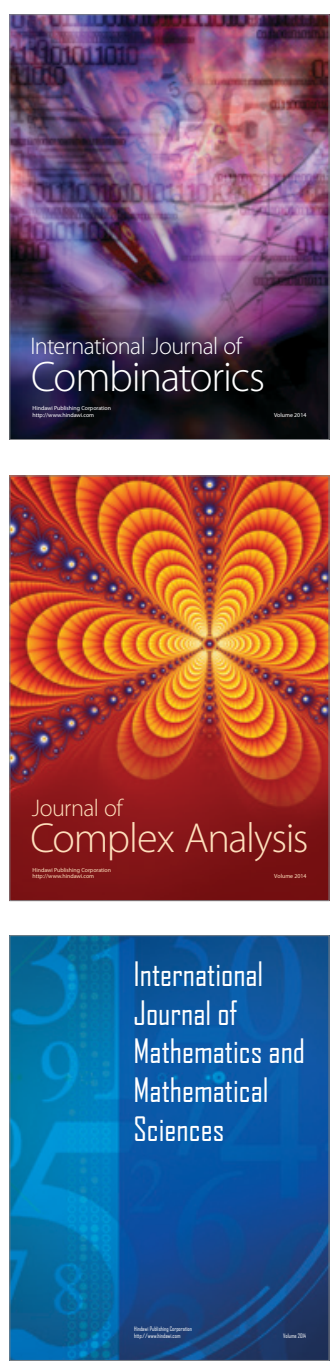
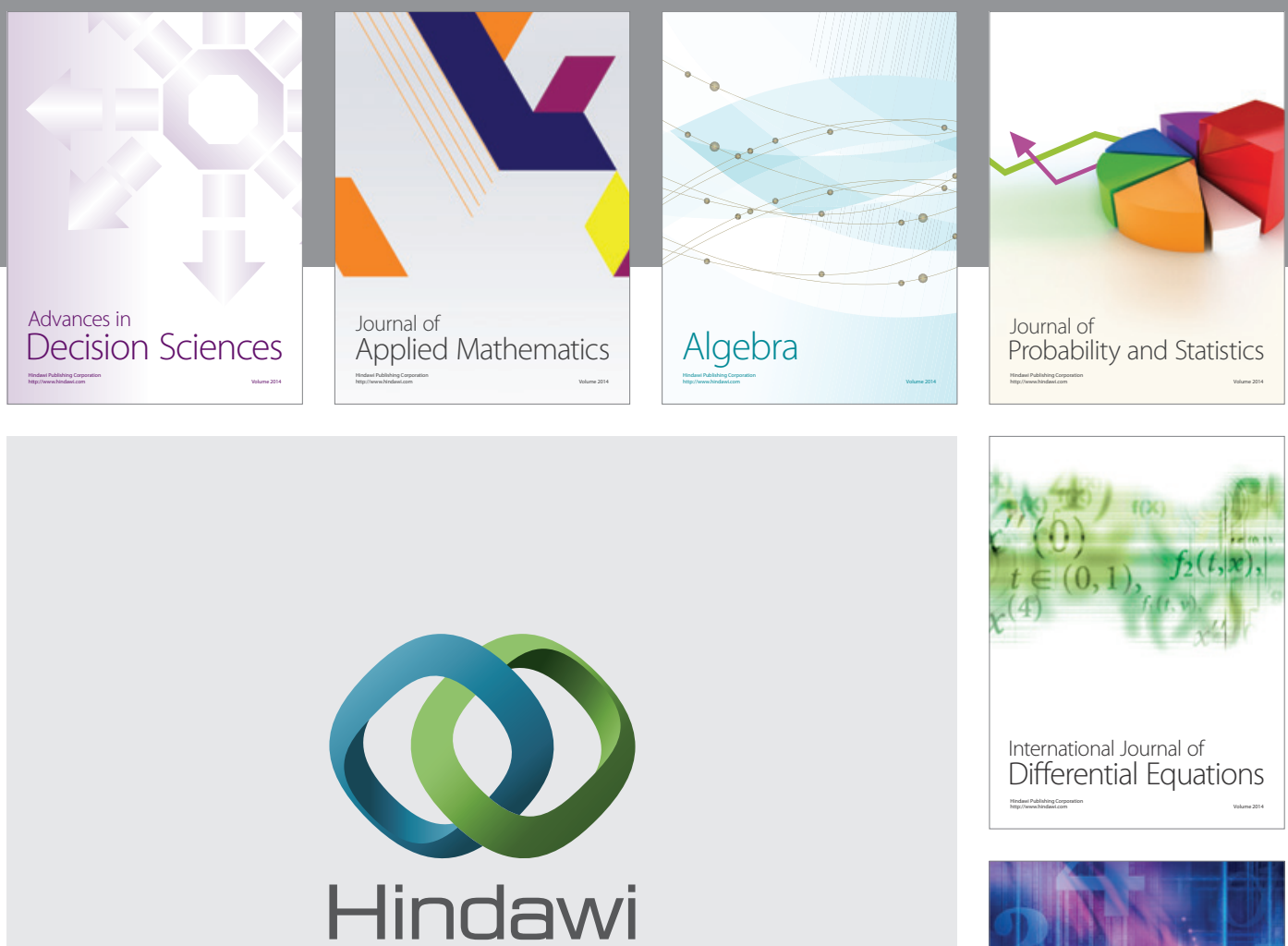

Submit your manuscripts at http://www.hindawi.com
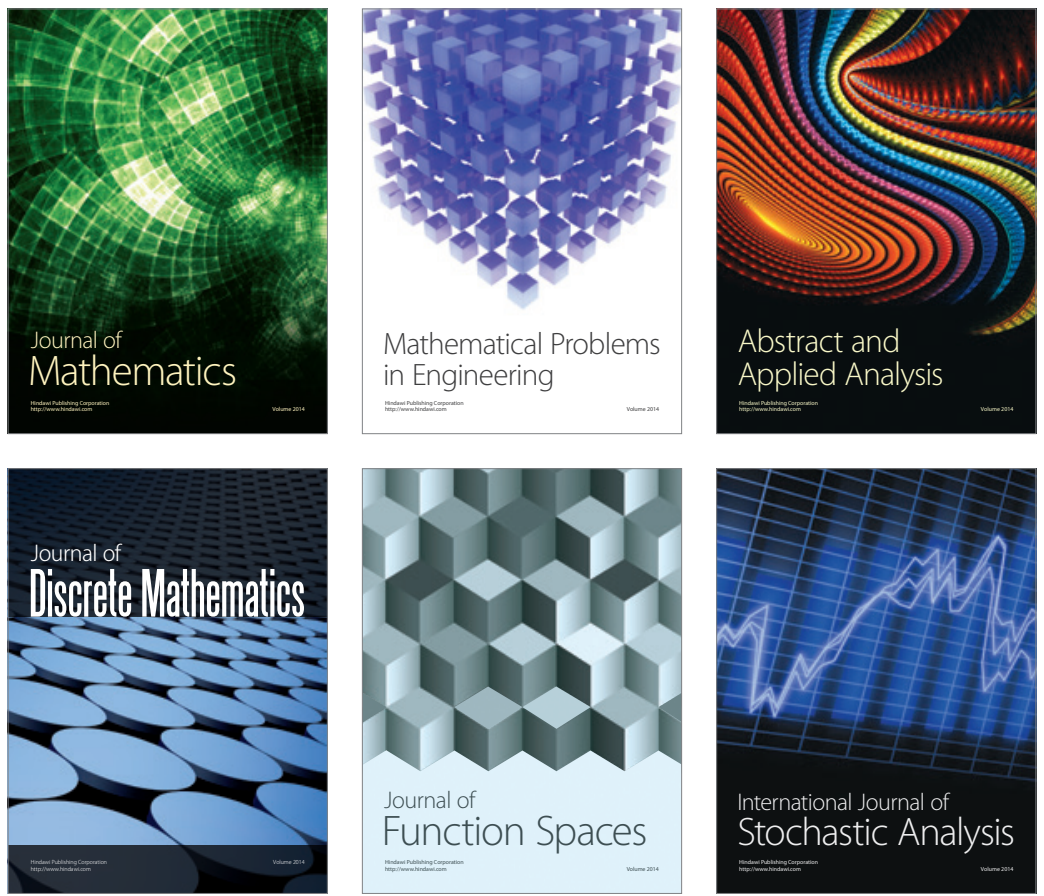

Journal of

Function Spaces

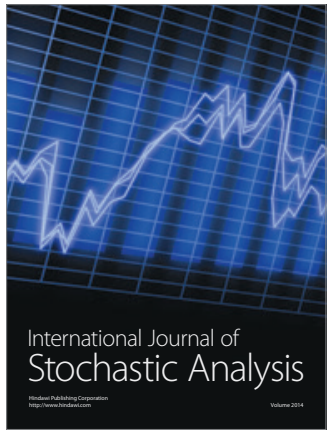

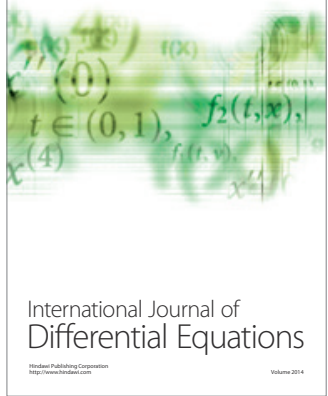
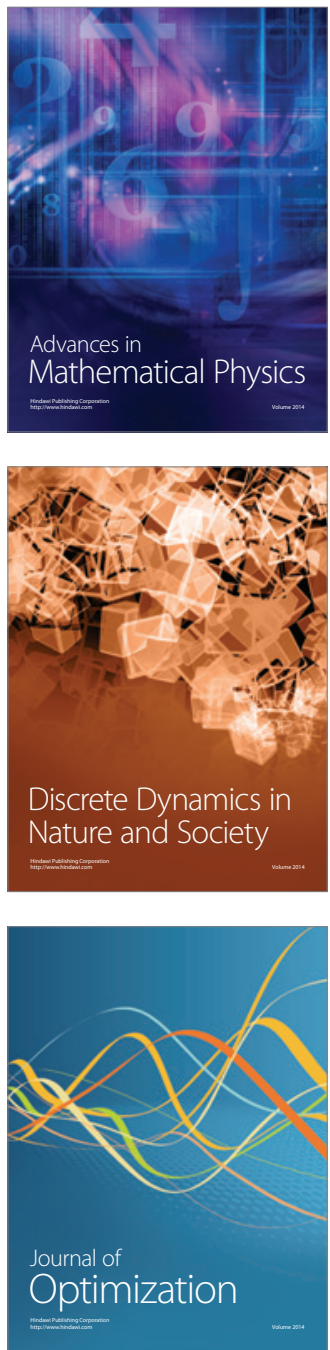